\title{
BMJ Open Public acceptability of non-pharmaceutical interventions to control a pandemic in the UK: a discrete choice experiment
}

\author{
Luis Enrique Loría-Rebolledo (D) , ${ }^{1}$ Mandy Ryan, ${ }^{1}$ Verity Watson, ${ }^{1}$ \\ Mesfin G Genie (D) , ${ }^{1}$ Ruben Andreas Sakowsky (D) , ${ }^{2}$ Daniel Powell (D) , ${ }^{3}$ \\ Shantini Paranjothy ${ }^{4}$
}

\begin{abstract}
To cite: Loría-Rebolledo LE, Ryan M, Watson V, et al. Public acceptability of nonpharmaceutical interventions to control a pandemic in the UK: a discrete choice experiment. BMJ Open 2022;12:e054155. doi:10.1136/ bmjopen-2021-054155
\end{abstract}

- Prepublication history and additional supplemental material for this paper are available online. To view these files, please visit the journal online (http://dx.doi.org/10.1136/ bmjopen-2021-054155).

Received 08 June 2021 Accepted 03 February 2022

Check for updates

(C) Author(s) (or their employer(s)) 2022. Re-use permitted under CC BY-NC. No commercial re-use. See rights and permissions. Published by BMJ.

${ }^{1}$ Health Economics Research Unit, University of Aberdeen, Aberdeen, UK

${ }^{2}$ Department of Medical Ethics and History of Medicine, University Medical Center Göttingen, Göttingen, Germany ${ }^{3}$ Health Psychology, University of Aberdeen, Aberdeen, UK

${ }^{4}$ Health Data Science Research Centre, University of Aberdeen, Aberdeen, UK

Correspondence to

Dr Luis Enrique Loría-Rebolledo luis.Ioria@abdn.ac.uk

\section{ABSTRACT}

Objective To understand how individuals trade off between features of non-pharmaceutical interventions (eg, lockdowns) to control a pandemic across the four nations of the UK.

Design A survey that included a discrete choice experiment. The survey design was informed using policy documents, social media analysis and input from remote think-aloud interviews with members of the public $(n=23)$. Setting A nationwide survey across the four nations of the UK using an online panel between 29 0ctober and 12 December 2020.

Participants Individuals who are over 18 years old. A total of 4120 adults completed the survey (1112 in England, 848 in Northern Ireland, 1143 in Scotland and 1098 in Wales).

Primary outcome measure Adult's preferences for, and trade-offs between, type of lockdown restrictions, length of lockdown, postponement of routine healthcare, excess deaths, impact on the ability to buy things and unemployment.

Results The majority of adults are willing to accept higher excess deaths if this means lockdowns that are less strict, shorter and do not postpone routine healthcare. On average, respondents in England were willing to accept a higher increase in excess deaths to have less strict lockdown restrictions introduced compared with Scotland, Northern Ireland and Wales, respectively. In all four countries, one out of five respondents were willing to reduce excess deaths at all costs.

Conclusions The majority of the UK population is willing to accept the increase in excess deaths associated with introducing less strict lockdown restrictions. The acceptability of different restriction scenarios varies according to the features of the lockdown and across countries. Governments can use information about tradeoff preferences to inform the introduction of different lockdown restriction levels and design compensation policies that maximise societal welfare.

\section{INTRODUCTION}

The COVID-19 pandemic has required countries worldwide to introduce nonpharmaceutical interventions to protect the health and well-being of their citizens. ${ }^{1}$ The majority of European and high-income

\section{Strengths and limitations of this study}

- This study provides a clear understanding of the trade-offs between restrictions and impacts of lockdowns on society, unlike existing data from opinion polls and citizens' panels.

- The results take into account the heterogeneity of UK nations and can be used to inform the introduction of different levels of lockdown restrictions in each nation.

- Given preferences are likely evolving, the dynamics of trade-offs for lockdown should be closely monitored

- We cannot disentangle between specific dimensions of lockdown restrictions that may affect preferences (eg, social activities, non-essential trips, school attendance and youth activities, non-essential businesses and outdoor activities).

nations have focused on reducing the reproduction number $(\mathrm{R})$ to less than 1 , thereby curtailing the epidemic spread of the virus and reducing the strain put on health systems. $^{2{ }^{3}}$ This strategy requires a number of non-pharmaceutical interventions such as enforced social distancing across all age groups, closing schools and non-essential businesses and a range of other social restrictions. ${ }^{4}$ This has led to local and nationwide lockdowns and other restrictions to control infection rates and excess deaths within geographically defined populations. ${ }^{5-7}$

Lockdowns have wider indirect impacts on health and well-being. Lockdown decisions require a careful balancing of the direct impacts on mortality caused by COVID-19 with the indirect wider health, social and economic impacts. ${ }^{8-11}$ Lockdown compliance will determine its effectiveness. Compliance is more likely to happen if policies are accepted by the public. Policies are more likely to be acceptable if the public's preferences are understood and the diversity of views is recognised. The WHO criteria for 
deciding whether to lift lockdown restrictions include the need for "Communities are fully educated, engaged and empowered to adjust to the "new norm" of everyday life, ${ }^{12}$ This criterion requires a better understanding of how the public responds to and values the trade-offs faced during and after pandemic. For example, is the public willing to accept a certain number of excess deaths to have restrictions eased?

Prior to the COVID-19 pandemic, there was limited evidence on how people think of lockdown policies in the UK. ${ }^{13}$ During the pandemic, public attitudes to government responses to the pandemic have been explored using opinion polls and qualitative studies. ${ }^{14-16}$ The Scottish Government and Bank of England established citizens' panels. ${ }^{17} 18$ These instruments offer insight into the views and concerns of the population. However, they provide no understanding of the trade-offs that individuals are willing to make. For example, the Scottish citizens' panel recommended that the Scottish Government should implement an elimination strategy, and where this is not feasible, should aim for maximum suppression of the virus, but did not state the cost of the restrictions that was acceptable to achieve this. Thus, we use a preference elicitation instrument tailored to quantify preferences, a discrete choice experiment (DCE), to provide new evidence on the acceptable number of excess deaths to the UK public when easing or tightening restrictions.

\section{METHODS}

\section{Study sample}

We conducted a cross-sectional survey among a sample of adults aged over 18 from across the four nations of the UK. The survey was implemented between 29 October and 12 December 2020. Respondents were recruited using an online survey research panel maintained by the company Qualtrics. The survey was piloted in early October 2020 $(\mathrm{n}=50$ per nation). Respondents were screened by the recruiting company using sex and age using quotas with the aim of achieving representativeness in each nation. The research company excluded respondents who completed the survey in less than half the median time of completion of the pilot stage of the survey $(14 \mathrm{~min})$.

\section{Discrete choice experiment}

Respondents self-completed an online survey that asked about the individual's experience during the COVID-19 pandemic, lockdowns that had occurred, any impacts on their healthcare, their spending ability and employment. The survey included a DCE, a choice-based survey that quantifies preferences for attributes (or features) of goods, services or policies. The DCE has its theoretical roots in Lancaster's theory of value and consumer theory. ${ }^{19}$ It assumes that goods or services (in this case, pandemic responses) can be described by attributes and the levels of these attributes. Each respondent faces a series of hypothetical scenarios (choice sets) composed of two or more alternatives. In each choice set, respondents are asked to
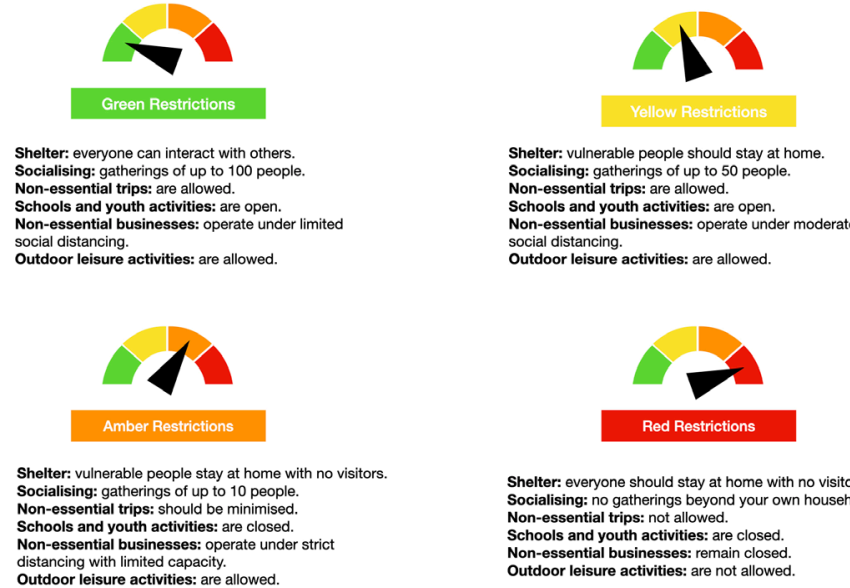

Figure 1 Lockdown levels for the 'type of lockdown' attribute.

choose their preferred scenario. The DCE assumes individuals consider all the information presented and make trade-offs.

Respondents completed a series of eight choice tasks based on the features of government restrictions. The hypothetical choice tasks focused on six features of government restrictions that describe different types of lockdown and their likely health and economic consequences. Features used to describe the type of lockdown were: restriction severity using a colour-based tier system (figure 1), length in weeks and postponement of routine healthcare procedures. Health consequences also included the number of excess deaths (we also report infection numbers as a complement based on the infection rate). ${ }^{20}$ We included two economic consequences: respondent's household's ability to buy things (personal impact) and the number of job losses (societal impact). See online supplemental table 1 for the features and associated levels. The features and levels were informed by policy documents, ${ }^{12}$ impacts of interventions that were implemented in response to COVID- $19,{ }^{4}$ literature on preferences for lockdown measures from previous pandemics ${ }^{212}$ and a social media analysis. A more detailed description of the development stage can be found in the study's published protocol. $^{23}$

Lockdown features and levels were combined into pairwise choice tasks using a D-efficient design. ${ }^{24} 25$ The design results in 24 tasks. Respondents were allocated randomly to one of the three survey versions, each with eight tasks. Respondents were asked to choose between two lockdown descriptions (figure 2). The order of the eight tasks was randomised for each respondent to minimise the ordering effects. ${ }^{26}$

\section{Patient and public involvement}

Adult members of the public were invited, using two targeted social media campaigns, to take part in the study development stage (see online supplemental figures $1-5)$. These engagements were used to create the survey's content and format, and to construct the framing of the 
Scenario 1
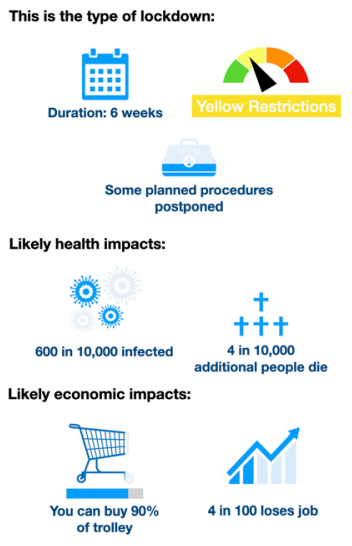

Scenario 2

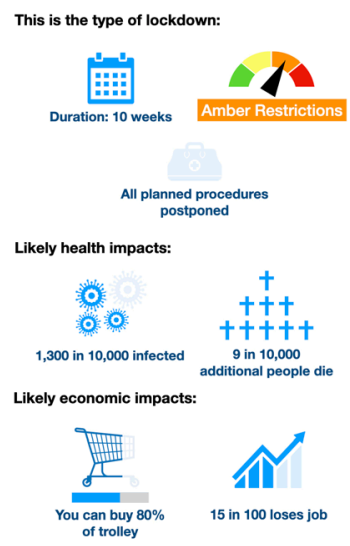

Figure 2 Example choice task as shown to respondents.

DCE features and levels (see online supplemental materials 1 and 2). Twenty-three think-aloud interviews were carried out between the months of June and August 2020. The outcome of each interview was used iteratively until saturation was achieved to make edits to the survey to ensure it captured the intended preferences, was understandable and minimised respondent burden. The study results will be disseminated to the wider public, with the help of the Stakeholder Advisory Group (SAG), using layperson summaries and multimedia content through mass media. Furthermore, the study's SAG, which includes a member of Scotland's Chief Scientist Office's Public Engagement Group, has been involved since its conception and provided insight into the research questions, overall design and dissemination strategy. Because of the study's ethical approvals, it is not possible for us to contact the members of the public who took part in the survey development stage, nor respondents of the main survey, to disseminate the results individually.

\section{Statistical analysis}

The devolved governments of the UK set their own lockdown policies; therefore, statistical analysis was conducted separately for each of the four devolved nations of the UK. The minimal sample size for the DCE given the eight tasks per respondent, a baseline choice probability of $50 \%$ (given there were two options in each choice set), an accuracy level of $90 \%$ and a confidence level of $95 \%$, using Louviere's formula for choice proportions, was 49 respondents. ${ }^{25}$ Given that we aimed to estimate preferences using flexible logit models, we aimed for a conservative size of 1000 per nation in the UK.

We focus on the trade-offs respondents were willing to make between the level of restriction and excess deaths. We first test if any respondents were unwilling to accept an increase in excess deaths for improvements in other features. This was defined as respondents who always chose the description with the lowest number of excess deaths. The response pattern for these respondents is shown in online supplemental table 2. We estimated a logit regression model to understand the characteristics of this group for each nation. Predictors included: sex, age, self-perceived health, number of children in household, household income quintile, whether they were asked to shield during previous lockdowns, had their main job impacted (furloughed, reduced hours or made redundant), had caring responsibilities and if they had seen their standard of living worsened during the COVID-19 pandemic.

We then analysed the choice tasks using an errorscomponent logit model, allowing for the panel structure of the data. ${ }^{27}$ Parameter estimates represented the effect of each feature on preferences. The ratio of estimates represents the trade-off between two features. Further, trade-offs between different features, when elicited in terms of a common denominator, can be added to estimate the overall trade-off for a particular lockdown scenario. When elicited in terms of excess deaths, these trade-offs indicate the maximum number of lives that need to be saved to introduce a hypothetical lockdown scenario. For example, how many lives would need to be saved (e.g. excess deaths avoided) when introducing a 4-week strict lockdown that cancels all non-COVID-19 healthcare procedures?

The difference in trade-offs between two lockdown scenarios can be interpreted as the maximum number of excess deaths that would be accepted if the more preferred scenario were introduced. To illustrate how these differences can inform policy, we assume that each nation faces a 4-week red level (see figure 1) restriction lockdown that postpones all non-COVID-19 healthcare procedures, and estimate the acceptable number of excess deaths to have this eased to less strict lockdown scenarios. Specifically, we compare easing to 12 different lockdowns made up of combinations of amber and yellow restrictions (figure 1) that vary in length between 8,10 and 12 weeks, and in whether they postpone healthcare services.

All logit models were estimated using maximum likelihood techniques using the statistical software $\mathrm{R}$ (V.3.6.3). SEs and CIs were computed using the delta method.

\section{RESULTS}

Respondents were not representative of the age and sex distributions of the four devolved nations; the response data were thus weighted using iterative proportional fitting. ${ }^{28}$ We also used matching to make our sample representative and found robust results. (Unweighted parameter estimate results are available in online supplemental material 1 ( p 14). Confirmatory results using matching are available from the authors.) A total of 4120 respondents completed the survey: 1112 in England, 848 in Northern Ireland, 1143 in Scotland and 1098 in Wales. Table 1 shows the sample descriptive characteristics across nations after weighting. 
Open access

Table 1 Characteristics associated with sample by nation*

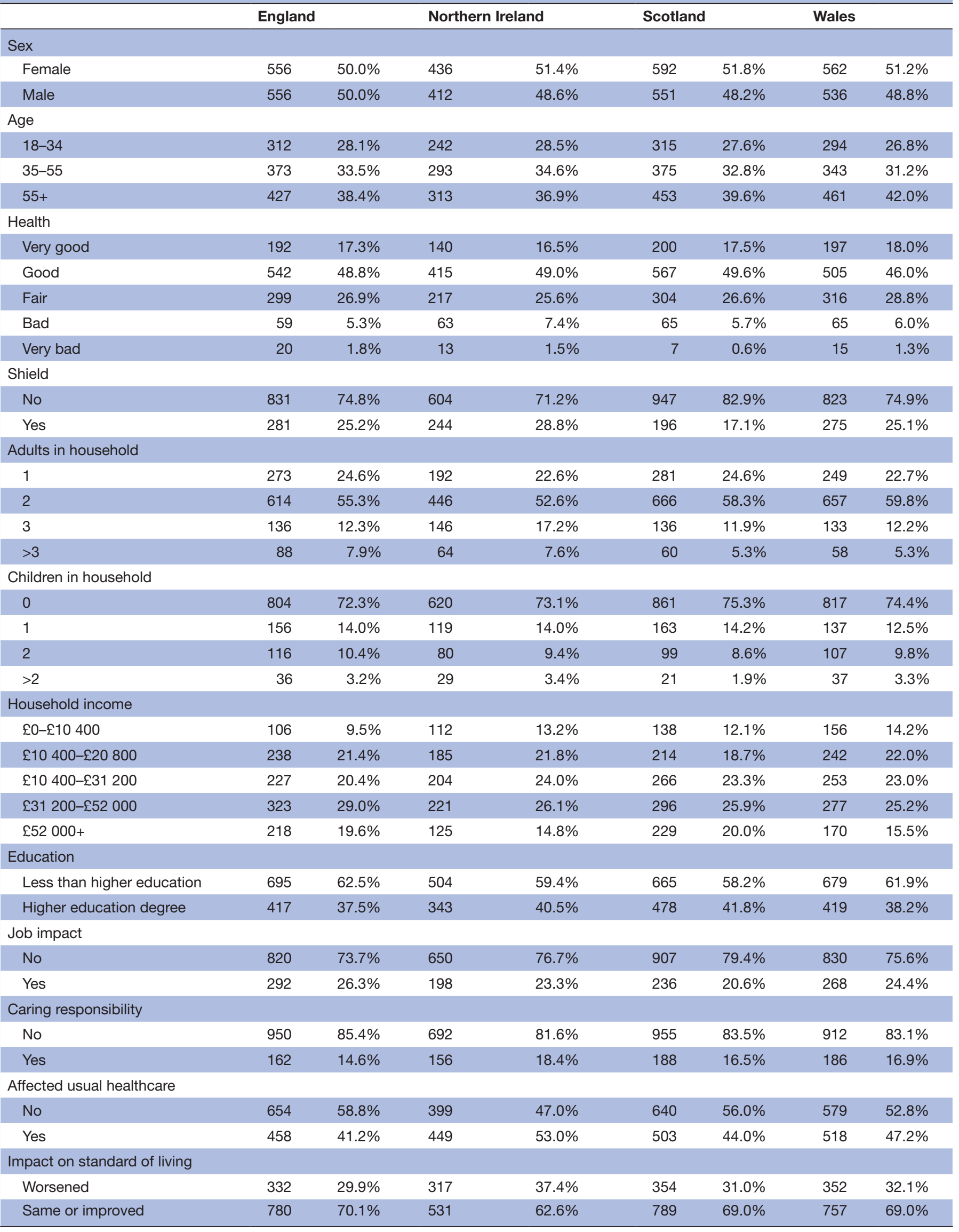




\begin{tabular}{lllll}
\hline & England & Northern Ireland & Scotland & Wales \\
\hline Total & 1112 & 848 & 1143 & 1098 \\
\hline
\end{tabular}

*Weighted frequencies.

The number of respondents who consistently chose the alternative with the least excess deaths was $225(20.2 \%)$ in England, 193 (22.8\%) in Northern Ireland, 262 (22.9\%) in Scotland and $247(22.5 \%)$ in Wales. Results from the logit model are shown in table 2. In England, none of the considered variables were associated with respondents always choosing the lowest number of excess deaths. In Northern Ireland, this response pattern was negatively associated with respondents who experienced an impact on employment (adjusted OR 0.58, 95\% CI 0.35 to 0.97 , $\mathrm{p}=0.04)$. In Scotland, this response pattern was also negatively associated with respondents who experienced an impact on employment $(0.62,95 \%$ CI 0.40 to $0.95, \mathrm{p}=0.03)$ and household income of $£ 20$ 800-£31 200 compared with the reference level of $£ 0-£ 10 \quad 400 \quad(0.54,95 \%$ CI 0.31 to $0.95, \mathrm{p}=0.03)$. Furthermore, this response pattern was positively associated with having a higher education degree (compared with less than higher education) (1.77, 95\% CI 1.28 to $2.45, \mathrm{p}<0.01)$ and fair self-reported health compared with very good $(1.82,95 \%$ CI 1.11 to 2.97, $\mathrm{p}=0.02)$. In Wales, this response pattern was negatively associated with age over 55 compared with 18-34 $(0.63,95 \%$ CI 0.40 to $0.98, \mathrm{p}=0.04)$, household income of $£ 10400-£ 20800(0.49,95 \%$ CI 0.29 to $0.83, \mathrm{p}=0.01)$, £20 800-£31 $200(0.57,95 \%$ CI 0.34 to $0.96, \mathrm{p}=0.04)$ and over $£ 52000(0.49,95 \%$ CI 0.79 to $1.56, \mathrm{p}=0.03)$ compared with $£ 0-£ 10400$. Univariate analyses for each factor are shown in online supplemental table 3 .

The preference parameter estimates and corresponding trade-offs in terms of excess deaths based on responses to the choice tasks are shown in table 3. Across the four nations, respondents prefer lockdowns with less strict restrictions (ie, green and yellow levels) to strict ones (ie, amber and red levels), shorter lockdowns, fewer excess deaths, fewer job losses and less impact on their ability to buy goods. In England, Northern Ireland and Scotland, respondents prefer no postponement of routine healthcare procedures (at the $10 \%$ level). The minimum number of lives (out of 10000 ) that need to be saved to accept a change in each of the lockdown features and consequences is shown in the marginal rates of substitution (MRS) column for each nation. Each MRS is calculated as the ratio of the parameter being valued to the negative of the parameter for excess deaths. For example, respondents in England would require a reduction in excess deaths of 2.08 lives per 10000 to accept a red lockdown $(-0.25 / 0.12)$ or alternatively they would be willing to accept 2.08 excess deaths per 10000 of the population to avoid a red lockdown.

Figure 3 shows the maximum acceptable excess deaths for easing restrictions from a further 4 -week red lockdown to the less strict lockdowns. The highest aversion to strict lockdowns is found in England, followed by Scotland, Northern Ireland and Wales, as seen by the higher number of acceptable excess deaths for lockdown easing. For example, the maximum number of acceptable deaths when easing to an 8-week yellow restriction with no healthcare postponement is 3.62 (95\% CI 2.67 to 4.58$)$ in England, 2.22 (95\% CI 1.21 to 3.24 ) in Northern Ireland, 2.41 (95\% CI 1.57 to 3.24$)$ in Scotland and $1.10(95 \%$ CI 0.18 to 2.02 ) in Wales. These rates equal 18 958; 361; 1265; and 323 excess deaths for each nation, respectively.

As expected, the maximum number of acceptable deaths is lower when moving to stricter (eg, amber over yellow) and longer lockdowns that postpone routine healthcare procedures. For example, the difference in the acceptable number of deaths between a 4-week red lockdown and a 12-week amber lockdown with healthcare postponement is 0.85 (95\% CI 0.03 to 1.67) in England and not statistically different from zero in Northern Ireland $\left(\chi^{2}=0.88, \mathrm{p}=0.35\right)$, Scotland $\left(\chi^{2}=1.84, \mathrm{p}=0.17\right)$ and Wales $\left(\chi^{2}=0.08, \mathrm{p}=0.77\right)$. This suggests that respondents in Northern Ireland, Scotland and Wales are indifferent between continuing with a further 4-week red restriction and easing to a 12-week amber restriction with healthcare postponement.

\section{DISCUSSION}

The elicitation of public values and trade-offs for different lockdown features can help guide government policies during a pandemic. We found evidence that four out of five respondents were willing to accept an increase in excess deaths for relaxations in lockdown restrictions. With the roll-out of pharmaceutical interventions and the increase in data available to model the impact of changes in restrictions, our results can help inform policy makers about what lockdown policies are acceptable given the estimated trade-offs. We found that respondents in England are the most averse to the introduction of short circuit-breaker-type lockdowns, thus accepting a higher number of excess deaths to avoid them. In contrast, these strict lockdowns were more palatable to respondents in Wales.

These insights are useful as UK governments consider the introduction of new restrictions in response to increased infections. More specifically, our model can be used to assess whether the expected health benefits in terms of a reduction in the number of excess deaths outweigh costs in terms of increased restrictions. As an example, modelling by Ferguson et al contended that a 1-week earlier strict lockdown in England during 


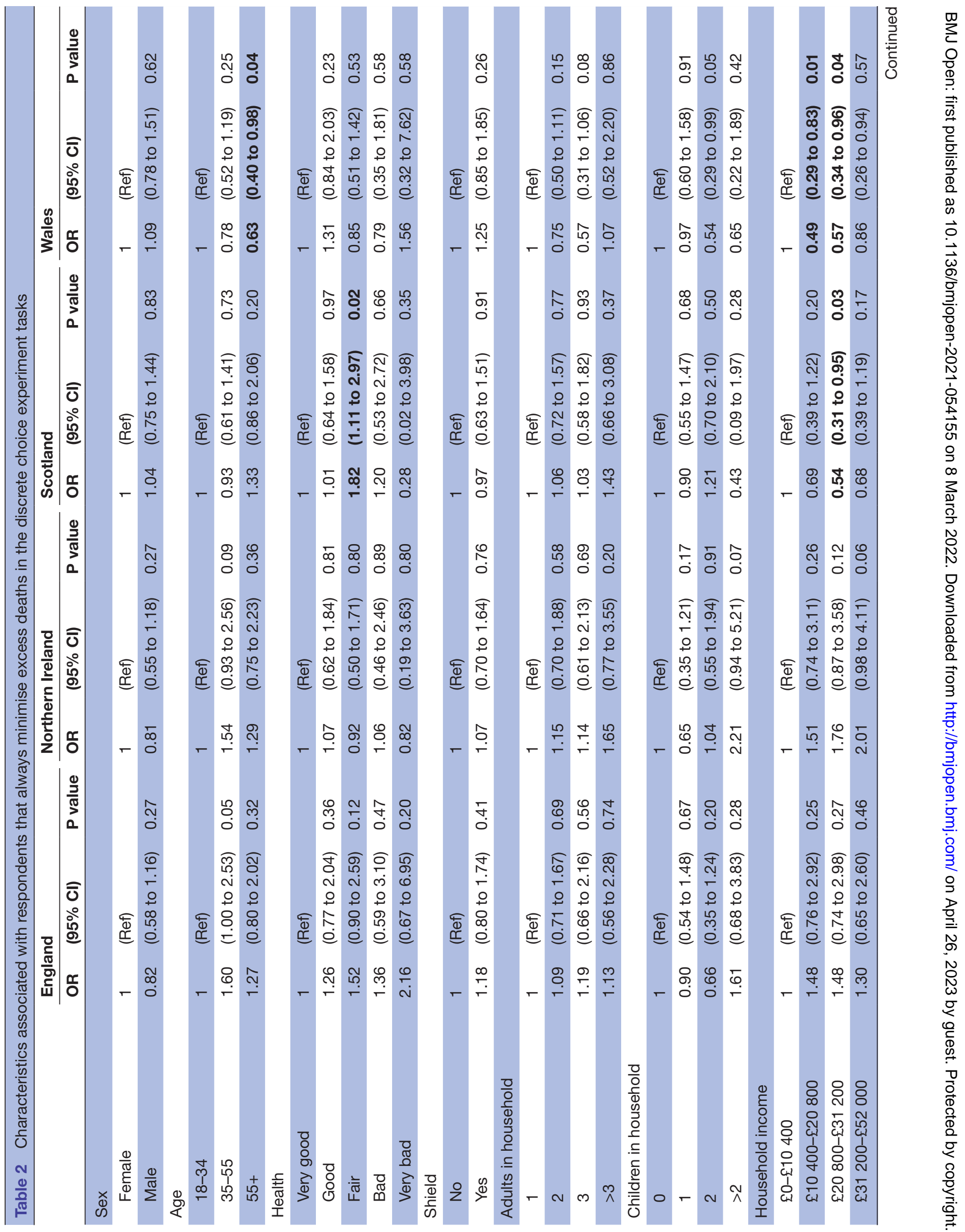




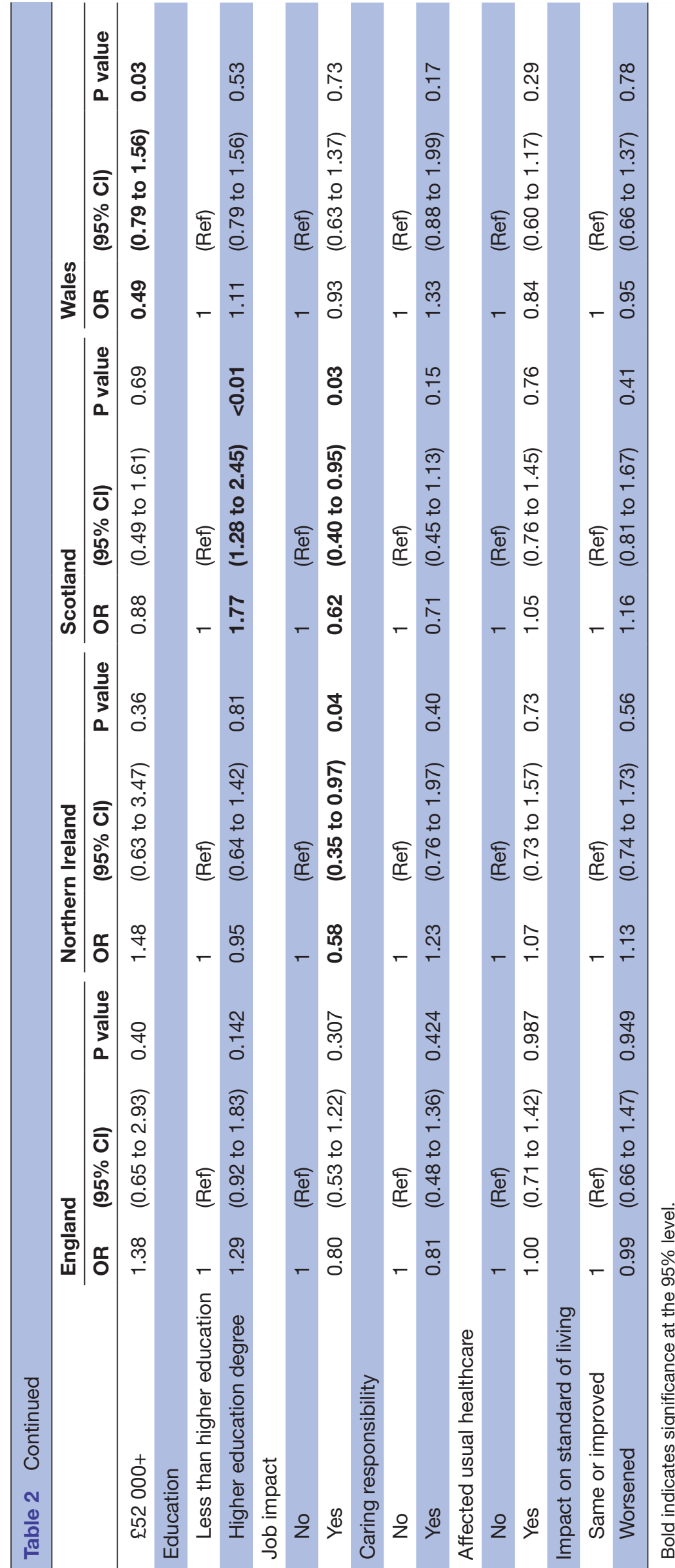

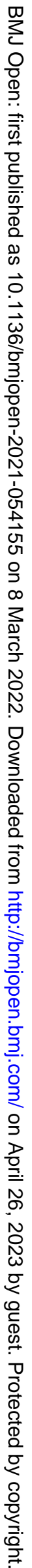




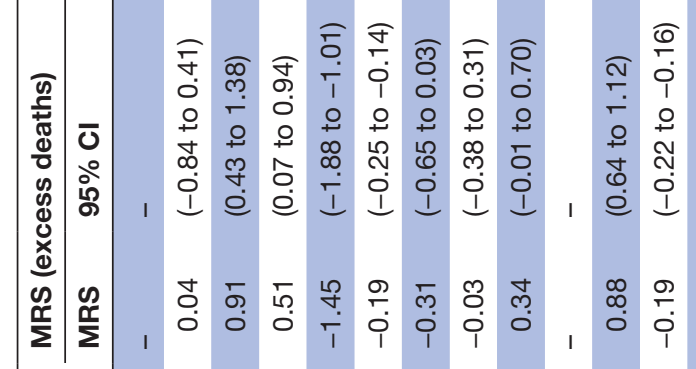

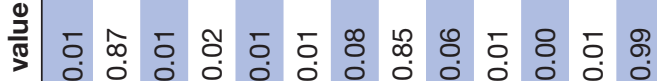

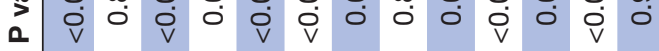

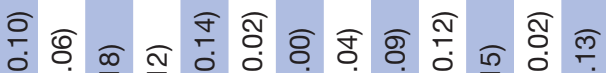

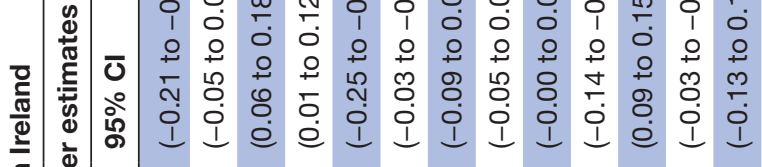

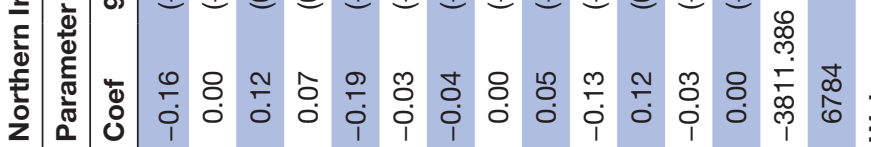
z

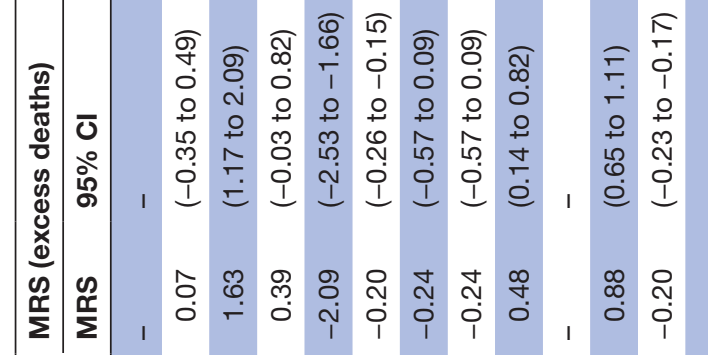

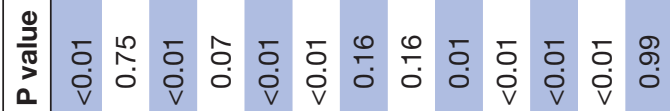

당 बิ

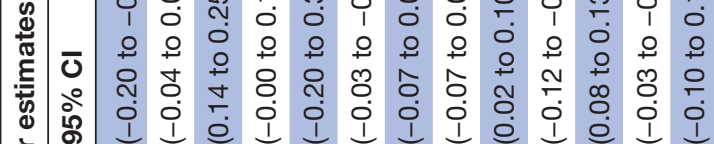

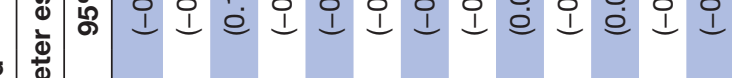

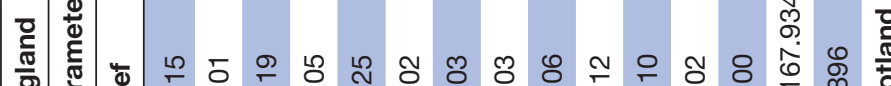

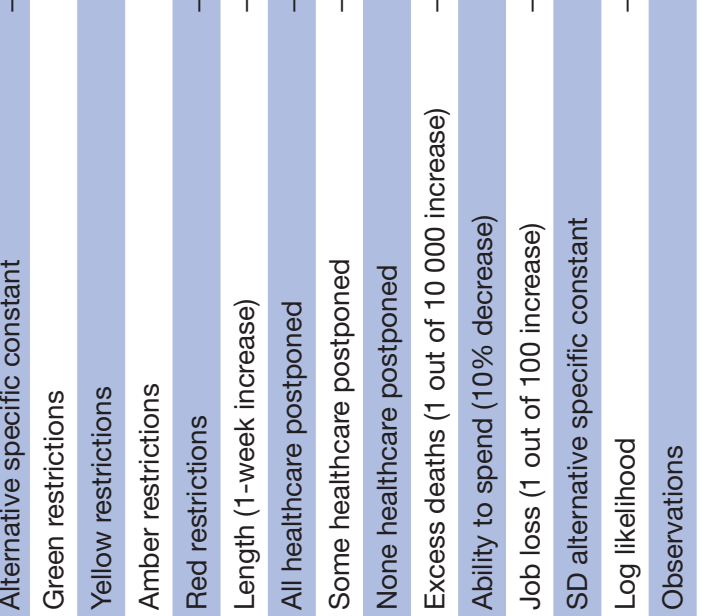

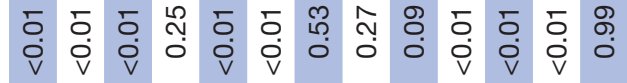

๙

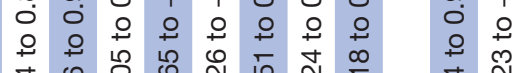

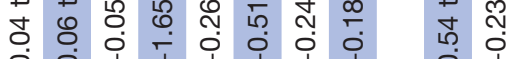

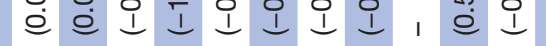

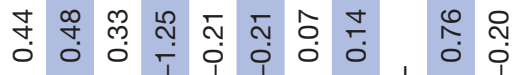

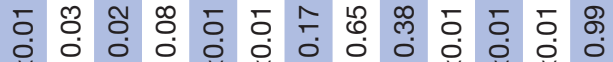

ঢ

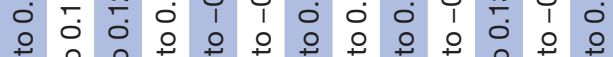

年

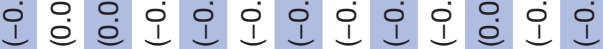

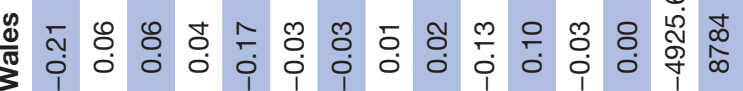

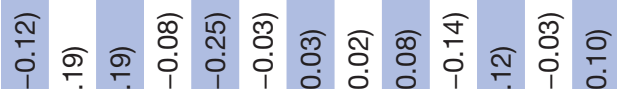

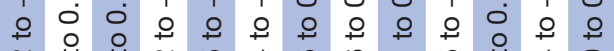

สำ

$\begin{array}{lllllll}1 & 0 & 0 & 1 & 1 & 1 & 1 \\ 0 & 1 & 1 & 1 & 0 & 1 & 1\end{array}$

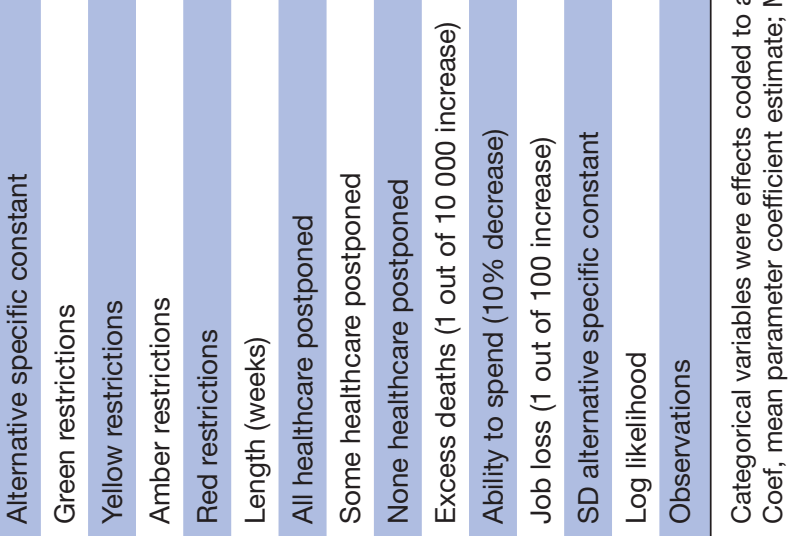



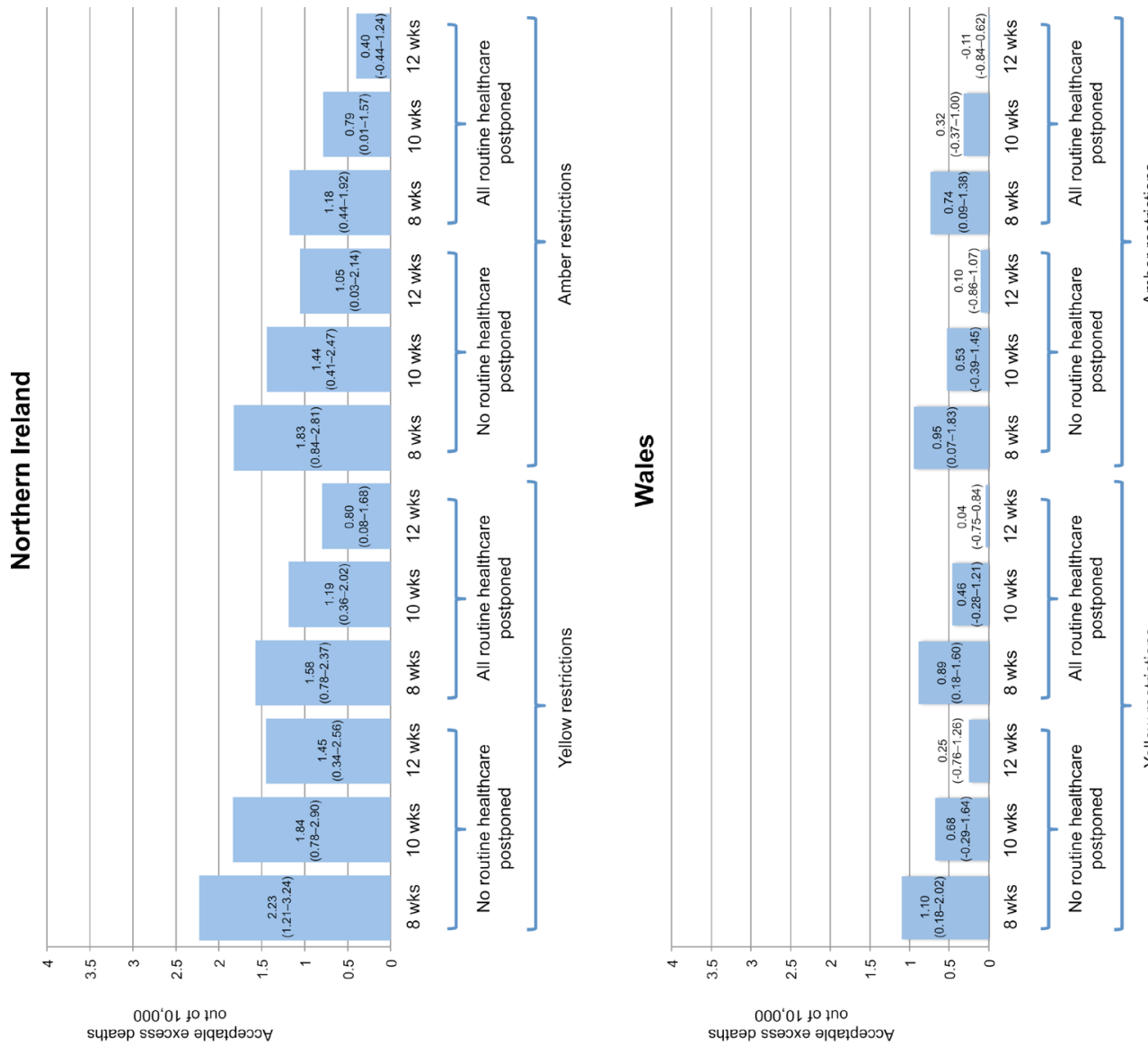

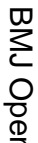

$\overrightarrow{\vec{B}}$

흠
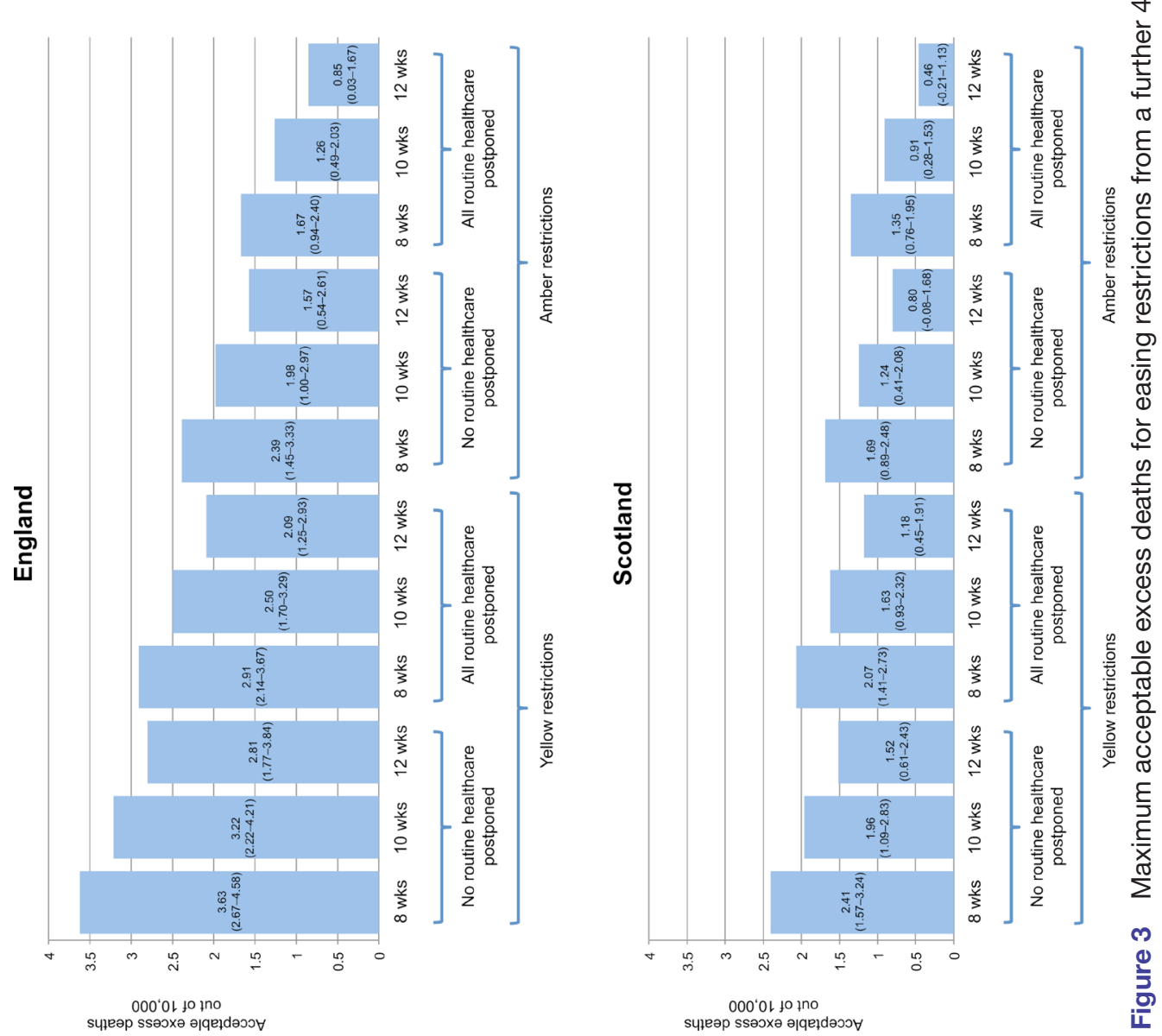
COVID-19's first wave would have saved 20000 lives. ${ }^{29}$ Our findings suggest that the number of acceptable deaths in England for a 1-week strict (red level restrictions) lockdown is 2.53 out of 10000 , or 14170 lives, which is less than the number of lives that would have been saved (see online supplemental material 1, p 16 for details). Thus, based on these results, the public's perception of the benefits of introducing an earlier lockdown would have outweighed the costs in terms of lockdown restrictions.

While we limited our analysis to consider acceptable excess deaths, a strength of our model is that it can be used to determine value in terms of other features included, that is, acceptable reductions in spending or job losses associated with a particular lockdown scenario. We found that respondents in Scotland were less sensitive to losses in their own spending ability compared with other nations. For example, the average acceptable loss in spending ability for a 4-week red level lockdown in Scotland is $49 \%$, while in England it is $36 \%$, Northern Ireland $29 \%$ and Wales $30 \%$. A detailed calculation of these trade-offs can be found in online supplemental material 1 (p 18). Thus, a targeted compensation instrument could target other economic consequences, such as joblessness, in Scotland and consumer-spending ability in the other nations.

We identified $20 \%$ of respondents as excess death minimisers, always choosing the option with the minimum number of deaths. This finding suggests that such respondents would accept any lockdown and economic costs to save lives. We found that no socioeconomic factors could consistently explain these findings. It is possible that respondents are trading, but the strong preference to minimise excess deaths results in the option with the lowest number of excess deaths being preferred. Another explanation is that this response pattern represents a decision heuristic for respondents to complete the tasks. We generated our choice sets using a D-efficient design, meaning that attribute levels approached orthogonality and there was a minimal overlap in choice sets. While increasing a design's statistical efficiency enables individual main effects to be estimated, there is a trade-off with cognitive efficiency since it also makes it more difficult for respondents to answer, potentially causing respondents to use heuristics. ${ }^{30}$ We note, however, that our design underwent extensive pretesting to ensure the tasks were not overly cognitively burdensome and enabled respondents to trade off across all attributes. Alternatively, the identified response pattern may relate to Tetlock's sacred values protection model, where sacred values are defined as any 'that a moral community implicitly or explicitly treats as possessing infinite or transcendental significance that precludes comparisons, trade-offs'. ${ }^{31} 32$

While we considered non-trading with respect to excess deaths, it is possible that respondents exhibited a nontrading behaviour with respect to other attributes. During the pandemic, discussions have taken place around whether the policy response should minimise job losses (which is likely less of a taboo trade-off), individual versus collective decision-making (eg, focusing on own ability to spend vs societal job losses) and the importance of individual freedom and civil liberties versus economic and health factors (eg, focusing on less restrictive lockdowns). ${ }^{33-37}$ Future research could investigate nontrading preference responses for all attributes, linking with socioeconomic characteristics and moral attitudes.

A potential limitation of our study is that individuals' preferences regarding the features of lockdowns may be evolving. Until March 2020, respondents would not have experienced a lockdown. We conducted our survey in October to December 2020, hence all respondents would have an experience of the first lockdown. The study was, however, conducted before the second lockdown. The dynamics of preferences and trade-offs for lockdown should be closely monitored. A further limitation is that the results are not necessarily transferable to other nations, although the methodology can be adapted for use in different populations. Recent DCE studies have also found the general public in Australia, ${ }^{38}$ the Netherlands, ${ }^{39}$ the USA $^{40}$ and France ${ }^{41}$ are willing to trade off specific health and non-health outcomes of lockdown interventions.

Our study did not look at the relative importance of the different dimensions of lockdown restrictions (shelter, socialising, non-essential trips, school and youth activities, non-essential businesses and outdoor activities). Future work could use a DCE to explore this; given current discussions around international travel, this dimension could be included. We focused on the public's preferences; future research could explore the preferences of policy makers, health professionals and groups especially affected by lockdown restrictions.

\section{CONCLUSIONS}

We have provided new insight into preferences for lockdown policies across the four UK nations using a DCE. The majority of respondents from all four devolved nations were willing to accept an increase in excess deaths for relaxation in lockdown restrictions. Respondents from England were more willing to accept an increase in excess deaths, followed by Scotland, Northern Ireland and Wales. Our model can also be used to estimate the reduction in excess deaths required to justify increasing lockdown restrictions. While we focused on excess deaths, trade-offs could also be estimated in terms of acceptable changes in spending power and job losses, as well as combinations of these features. Such analysis will help identify which levers best support lockdown strategies while maintaining public confidence and maximising compliance.

Twitter Luis Enrique Loría-Rebolledo @luisloria and Mesfin G Genie @mesfin_genie

Acknowledgements We want to thank the members of the public who took part in the think-aloud interviews as part of the study development stage. We thank the members of the Stakeholder Advisory Group for their continued involvement in the research study. We also thank Dr Dwayne Boyers for his internal review of the study protocol and Professor Vikki Entwistle for comments on the protocol. 
Contributors MR, VW, MGG, RAS and LEL-R conceptualised the study, contributed to the overall design of the survey experiment and contributed to the interpretation of the data. MGG and RAS carried out the think-aloud interviews as part of the developmental work. LEL-R undertook the analysis, including the $\mathrm{R}$ programming of the statistical models. MR reviewed the statistical model and contributed to the analysis of the data. SP and DP contributed comments to the development of the protocol, and discussion of public health implications, and helped shape the overall interpretation. LEL-R is the study's guarantor and accepts full responsibility for the work and/or the conduct of the study, had access to the data, and controlled the decision to publish. All authors approved the final manuscript. All authors had access to all the data, contributed to the writing of the paper and had final responsibility for the decision to submit for publication.

Funding This study was funded by the Health Economics Research Unit, the University of Aberdeen, and the Chief Scientist Office of the Scottish Government Health and Social Care Directorates.

\section{Competing interests None declared.}

Patient consent for publication Not applicable.

Ethics approval This study involves human participants and was approved by the University of Aberdeen's College of Life Sciences and Medicine Ethical Review Board (reference CERB/2020/6/1974). Participants gave informed consent to participate in the study before taking part.

Provenance and peer review Not commissioned; externally peer reviewed.

Data availability statement Data are available upon reasonable request. Anonymised cross-sectional data from the analysis can be made available by the corresponding author after the authors' review of reasonable requests. The published protocol can be found at http://dx.doi.org/10.1136/bmjopen-2020043477.

Supplemental material This content has been supplied by the author(s). It has not been vetted by BMJ Publishing Group Limited (BMJ) and may not have been peer-reviewed. Any opinions or recommendations discussed are solely those of the author(s) and are not endorsed by BMJ. BMJ disclaims all liability and responsibility arising from any reliance placed on the content. Where the content includes any translated material, BMJ does not warrant the accuracy and reliability of the translations (including but not limited to local regulations, clinical guidelines, terminology, drug names and drug dosages), and is not responsible for any error and/or omissions arising from translation and adaptation or otherwise.

Open access This is an open access article distributed in accordance with the Creative Commons Attribution Non Commercial (CC BY-NC 4.0) license, which permits others to distribute, remix, adapt, build upon this work non-commercially, and license their derivative works on different terms, provided the original work is properly cited, appropriate credit is given, any changes made indicated, and the use is non-commercial. See: http://creativecommons.org/licenses/by-nc/4.0/.

\section{ORCID iDs}

Luis Enrique Loría-Rebolledo http://orcid.org/0000-0002-1391-6478

Mesfin G Genie http://orcid.org/0000-0002-1744-4666

Ruben Andreas Sakowsky http://orcid.org/0000-0002-6893-9220

Daniel Powell http://orcid.org/0000-0003-4995-6057

\section{REFERENCES}

1 Eubank S, Eckstrand I, Lewis B, et al. Commentary on Ferguson, et al., "Impact of Non-pharmaceutical Interventions (NPIs) to Reduce COVID-19 Mortality and Healthcare Demand". Bull Math Biol 2020;82:52.

2 Hale T, Angrist N, Goldszmidt R, et al. A global panel database of pandemic policies (Oxford COVID-19 government response Tracker). Nat Hum Behav 2021;5:529-38.

3 Mahtani KR, Heneghan C, Aronson JK. What is the evidence for social distancing during global pandemics? A rapid summary of current knowledge. The Centre for Evidence-Based Medicine, 2020. Available: https://www.cebm.net/covid-19/what-is-the-evidence-forsocial-distancing-during-global-pandemics/accessed

4 Centre for Disease Control and Prevention. Social distancing, 2020. Available: https://www.cdc.gov/coronavirus/2019-ncov/preventgetting-sick/social-distancing.html [Accessed April 2020].

5 Mahase E. Covid-19: UK starts social distancing after new model points to 260000 potential deaths. BMJ 2020;368:m1089.

6 Scientific Advisory Group for Emergencies. Scientific evidence supporting the government response to coronavirus (COVID-19),
2021. Available: https://www.gov.uk/government/collections/ scientific-evidence-supporting-the-government-response-tocoronavirus-covid-19\#spi-b-background-papers [Accessed March, 2021].

7 Colbourn T. COVID-19: extending or relaxing distancing control measures. Lancet Public Health 2020;5:e236-7.

8 Venkatesh A, Edirappuli S. Social distancing in covid-19: what are the mental health implications? BMJ 2020;10:m1379.

9 Koren M, Petö R. Business disruptions from social distancing. PLoS One 2020;15:e0239113.

10 Coibion O, Gorodnichenko Y, Weber M. The cost of the covid-19 crisis: Lockdowns, macroeconomic expectations, and consumer spending. National Bureau of Economic Research 2020:w27141.

11 Chetty R, Friedman J, Hendren N. How did covid-19 and stabilisation policies affect spending and employment? a new real-time economic tracker based on private sector data. NBER working paper 2020:w27431.

12 World Health Organization. Coronavirus disease (COVID-19) pandemic, 2021. Available: https://www.who.int/emergencies/ diseases/novel-coronavirus-2019 [Accessed March, 2020].

13 Cabinet Office. Overarching government strategy to respond to pandemic influenza - analysis of scientific evidence base. Available: https://assets.publishing.service.gov.uk/government/uploads/ system/uploads/attachment_data/file/61968/flu_pandemic_science_ paper1.pdf [Accessed April, 2021].

14 Ipsos MORI. Public opinion on the COVID-19 coronavirus pandemic, 2021. Available: https://www.ipsos.com/ipsos-mori/ en-uk/public-opinion-covid-19-coronavirus-pandemic [Accessed February, 2021].

15 University College London. Understanding the psychological and social impact of the pandemic, 2021. Available: https://www. covidsocialstudy.org/ [Accessed February, 2021].

16 Williams SN, Armitage CJ, Tampe T, et al. Public perceptions and experiences of social distancing and social isolation during the COVID-19 pandemic: a UK-based focus group study. BMJ Open 2020;10:e039334.

17 Bank of England. The UK economy: Insights from the Bank of England's Citizens' Panels, 2020. Available: https://www. bankofengland.co.uk/get-involved/citizens-panels/the-uk-economyinsights-from-the-bank-of-englands-citizens-panels [Accessed February, 2021].

18 The Scottish Parliament. Covid-19: citizens' panel, 2021. Available: https://www.parliament.scot/parliamentarybusiness/ CurrentCommittees/116947.aspx [Accessed March, 2021].

19 Lancaster KJ. A new approach to consumer theory. J Polit Econ 1966;74:132-57.

20 Meyerowitz-Katz G, Merone L. A systematic review and metaanalysis of published research data on COVID-19 infection fatality rates. Int J Infect Dis 2020;101:138-48.

21 Cook AR, Zhao X, Chen MIC, et al. Public preferences for interventions to prevent emerging infectious disease threats: a discrete choice experiment. BMJ Open 2018;8:e017355.

22 Johnson J, Howard K, Wilson A, et al. Public preferences for one health approaches to emerging infectious diseases: a discrete choice experiment. Soc Sci Med 2019;228:164-71.

23 Genie MG, Loría-Rebolledo LE, Paranjothy S, et al. Understanding public preferences and trade-offs for government responses during a pandemic: a protocol for a discrete choice experiment in the UK. BMJ Open 2020;10:e043477.

24 Bech M, Kjaer T, Lauridsen J. Does the number of choice sets matter? Results from a web survey applying a discrete choice experiment. Health Econ 2011;20:273-86.

25 Rose JM, Bliemer MCJ. Sample size requirements for stated choice experiments. Transportation 2013;40:1021-41.

26 Ryan M, Krucien N, Hermens F. The eyes have it: using eye tracking to inform information processing strategies in multi-attributes choices. Health Econ 2018;27:709-21.

27 Lancsar E, Fiebig DG, Hole AR. Discrete choice experiments: a guide to model specification, estimation and software. Pharmacoeconomics 2017;35:697-716.

28 Kolenikov S. Calibrating survey data using iterative proportional fitting (raking). Stata J 2014;14:22-59.

29 Ferguson N, Laydon D, Nedjati Gilani G. Report 9: impact of nonpharmaceutical interventions (NPIs) to reduce COVID19 mortality and healthcare demand 2020.

30 Flynn TN, Bilger M, Malhotra C, et al. Are efficient designs used in discrete choice experiments too difficult for some respondents? A case study eliciting preferences for end-of-life care. Pharmacoeconomics 2016;34:273-84.

31 Fiske AP, Tetlock PE. Taboo Trade-offs: reactions to transactions that Transgress the spheres of justice. Polit Psychol 1997;18:255-97. 
32 Tetlock PE, Kristel OV, Elson SB, et al. The psychology of the unthinkable: taboo trade-offs, forbidden base rates, and heretical counterfactuals. J Pers Soc Psychol 2000;78:853-70.

33 UK Cabinet Office. COVID-19 response: summer 2021. author, 2021. Available: https://www.gov.uk/government/publications/covid-19response-summer-2021-roadmap/covid-19-response-summer-2021 [Accessed November, 2021].

34 Sekalala S, Forman L, Habibi R, et al. Health and human rights are inextricably linked in the COVID-19 response. BMJ Glob Health 2020;5:e003359.

35 Coibion O, Gorodnichenko Y, Weber M. The cost of the covid-19 crisis: Lockdowns, macroeconomic expectations, and consumer spending (No. w27141. National Bureau of Economic Research, 2020.

36 Blanchard O, Philippon T, Pisani-Ferry J. A new policy toolkit is needed as countries exit COVID-19 lockdowns. Bruegel, 2020.

37 Martin A, Markhvida M, Hallegatte S, et al. Socio-Economic impacts of COVID-19 on household consumption and poverty. Econ Disaster Clim Chang 2020;4:453-79.
38 Manipis K, Street D, Cronin P, et al. Exploring the trade-off between economic and health outcomes during a pandemic: a discrete choice experiment of lockdown policies in Australia. Patient 2021;14:359-71.

39 Chorus C, Sandorf ED, Mouter N. Diabolical dilemmas of COVID-19: an empirical study into Dutch Society's trade-offs between health impacts and other effects of the lockdown. PLoS One 2020;15:e0238683.

40 Reed S, Gonzalez JM, Johnson FR. Willingness to accept tradeoffs among COVID-19 cases, social-distancing restrictions, and economic impact: a nationwide US study. Value Health 2020;23:1438-43.

41 Sicsic J, Blondel S, Chyderiotis S. Preferences for COVID-19 epidemic control measures among French adults: a discrete choice experiment. MedRvix 2021.

42 Bech M, Gyrd-Hansen D. Effects coding in discrete choice experiments. Health Econ 2005;14:1079-83. 